\title{
Maintenance des ouvrages en terre sur le réseau ferré national
}

V. TALFUMIÈRE SNCF

6, avenue

François-Mitterrand 93574 La Plaine Saint-Denis vincent.talfumiere@sncf.fr

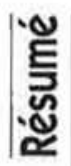

Le réseau ferroviaire français comporte un important patrimoine d'ouvrages en terre, dont l'état et le comportement peuvent influer notablement sur la régularité et la sécurité des circulations. Une organisation et une méthodologie spécifiques ont été mises en œuvre par la SNCF pour assurer, pour le compte de RFF, propriétaire du réseau, la surveillance, l'entretien et la régénération des ouvrages en terre. La particularité du patrimoine des ouvrages en terre est sa grande diversité tant en nature d'ouvrage (constitution, géométrie, etc.) que du point de vue environnement (plaines inondables, versants rocheux, etc.). Pour chaque type d'ouvrage, des désordres particuliers peuvent être observés. De plus, les ouvrages en terre ferroviaires se distinguent par des contraintes spécifiques. La SNCF réalise pour le compte de RFF cette surveillance spécifique visant à détecter au plus tôt les indices de désordres pour pouvoir limiter ainsi les conséquences sur les circulations ferroviaires. Les désordres détectés peuvent nécessiter deux types de traitement : des travaux d'entretien permettant d'intervenir préventivement avant l'apparition de désordres plus conséquents, des travaux confortatifs pour stabiliser l'ouvrage ou des parades pour se protéger des désordres dont l'origine se situe en dehors des emprises dans l'environnement.

Mots-clés : ouvrages en terre, maintenance, surveillance des risques, voies ferrées.

\section{Maintenance of ground works on the french railroad network}

The French railroad network contains an important patrimony of ground works whose state and behaviour can significantly affect the regularity and the safety of traffic. A specific organization and methodology were implemented by the SNCF to ensure, for RFF, the owner of the network, the monitoring, the maintenance and the regeneration of the ground works. The peculiarity of the ground works patrimony is its big variety from the points of view of both kind of works (constitution, geometry, etc.) and environment (flooding plains, rocky hillsides, etc.). For each kind of ground work, particular disorders can be observed. Besides, the railroad ground works are characterised by specific constraints. The SNCF performs for RFF this specific monitoring to detect the disorders evidences as soon as possible so as to limit the consequences on the rail traffics. The detected disorders can require two kinds of treatment: maintenance works allowing preventive intervention before the appearance of more consequent disorders, and so reinforcement works to stabilize the structure or parades to protect ground works from disorders whose origin is situated outside the property.

Key words: ground works, maintenance, risks monitoring, railroads. 
1

\section{Introduction}

Les ouvrages en terre (OT) sont une des composantes du patrimoine du réseau ferroviaire français ; depuis 1997, date de sa création, Réseau Ferré de France (RFF) est propriétaire et donc maître d'ouvrage (MOA) de l'infrastructure ferroviaire (ouvrages en terre, ouvrages d'art, rails, caténaires, cours de gare, etc.). Elle délègue la gestion de ce patrimoine à la SNCF en tant que gérant de l'infrastructure délégué (GID).

Le réseau ferroviaire français comprend environ $32000 \mathrm{~km}$ de lignes. Si l'on retire les ouvrages d'art (viaducs, ponts, tunnels), soit environ $1000 \mathrm{~km}$, il reste $31000 \mathrm{~km}$ de plate-forme ferroviaire qui constitue le patrimoine des ouvrages en terre du réseau ferré français.

Par ouvrage en terre, on entend tout ouvrage, résultant de la modification par terrassement d'un site pour la création d'une plate-forme ferroviaire. Ces ouvrages peuvent être rasants (au niveau du terrain naturel) ou établis en remblai ou en déblai, parfois de grande hauteur (jusqu'à $50 \mathrm{~m}$ pour quelques ouvrages très spécifiques). On estime le nombre total d'ouvrages en terre à environ 100000 unités. Ces ouvrages ont été créés pour la plupart il y a plus de 100 à 150 ans. La particularité du patrimoine des ouvrages en terre est sa grande diversité tant en nature d'ouvrage (constitution, géométrie, etc.) que du point de vue de leur environnement (plaines inondables, versants rocheux, etc.). On distingue les ouvrages en terre meuble, constitués de matériaux assimilés à des sols, des ouvrages en terre rocheux dont les matériaux sont indurés. Pour chaque type d'ouvrage, des désordres particuliers peuvent être observés. Par ailleurs, les ouvrages en terre ferroviaires se distinguent par :

- les contraintes propres aux circulations ferroviaires (maintien du trafic);

- les difficultés d'accès ;

- la nécessité de garantir la sécurité des biens et des personnes transportées (contrat entre la SNCF et le voyageur) ;

- l'utilisation de méthodes de confortement et de prescriptions d'exécution adaptées au contexte spécifique de l'exploitation ferroviaire.

\section{Désordres et incidents}

Les ouvrages en terre sont susceptibles de voir leur condition de stabilité s'altérer avec le temps, à cause de facteurs internes ou externes, qui peuvent entraîner des déformations irréversibles avec détérioration de la géométrie de la voie ferrée et/ou des ruptures plus ou moins brutales dans certains cas. Beaucoup ont fait l'objet de confortements dès leur construction ou dans le courant de leur vie : ces confortements vieillissent et deviennent moins efficaces, l'augmentation des trafics et des charges sur certains axes contribuant à ce vieillissement. Par ailleurs, les voies ferrées sont en plus exposées à des désordres dont l'origine provient de leur environnement (par exemple : versant rocheux, environnement karstique ou modifications faites de la main de l'homme).

Lorsque le désordre est brutal, on parle d'incident. Chaque année depuis près de 30 ans, le nombre d'incidents est recensé par les services gérants de l'infrastructure (branche infra à qui RFF délègue la gestion du patrimoine). On compte entre 150 et 450 incidents par an, de gravité plus ou moins importante, certains affectant les voies, allant du simple ravinement à l'éboulement rocheux ou la rupture d'un remblai. Le nombre d'incidents varie de façon importante car il est lié aux intempéries et à la pluviométrie (orage brutal ou longue période de pluie de durée supérieure à une semaine par exemple, Figure 1). On remarquera que l'année 2001, beaucoup plus pluvieuse entre février et mai, a vu l'apparition de plus de 250 incidents en 4 mois.

On distingue plusieurs catégories d'incidents suivant le type et la nature de l'ouvrage :

- les glissements de remblai ou de déblai (Figs. 2 et 3 ) ;

- les coulées boueuses;

- les fontis (Fig. 4) ;

- les chutes de blocs (Fig. 5).

La sécurité et la régularité des circulations ferroviaires nécessitent donc une surveillance et un suivi de l'évolution des ouvrages pouvant déboucher en cas de désordre sur des travaux d'entretien ou de confortement visant à maintenir ou améliorer leur stabilité. Ces interventions peuvent être préventives ou curatives.

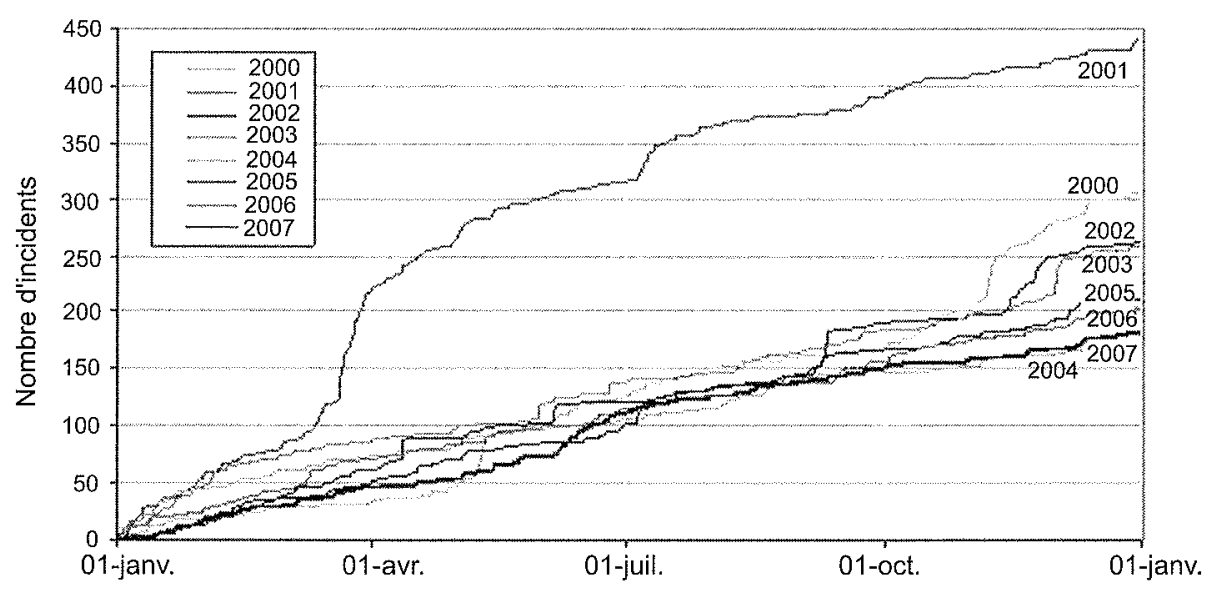

FG. 1 Cumulée du nombre d'incidents ouvrages en terre sur le réseau ferré français pour les années 2000-2007.

Summary of the number of incidents concerning ground works on the French railroad network for years 2000-2007. 


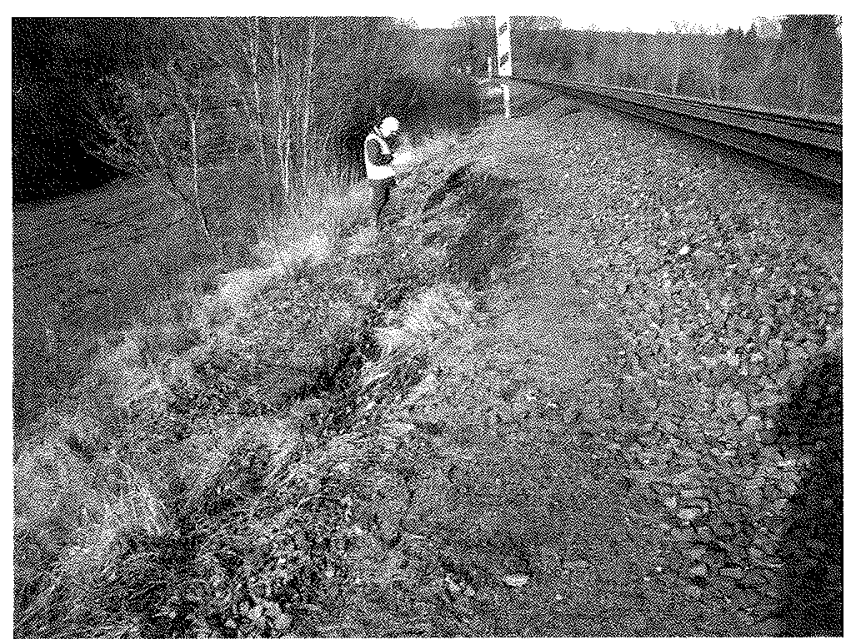

H6. 2 Incident en remblai de type glissement. Embankment incident, sliding.

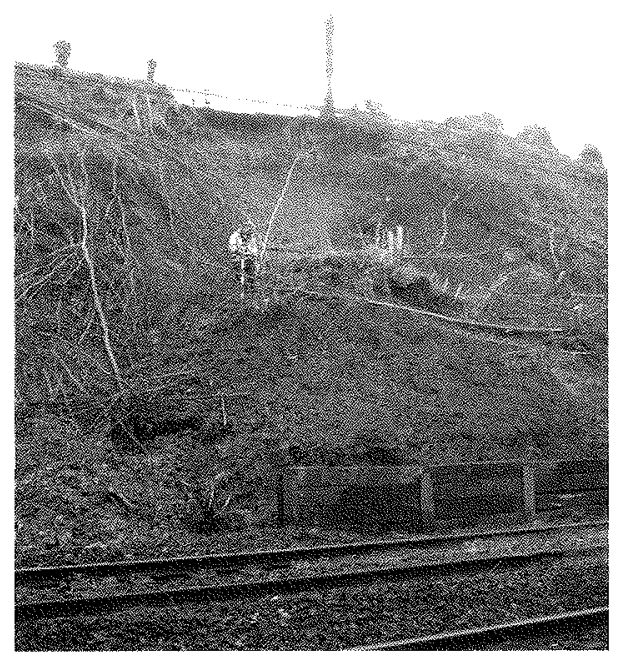

Ha. 3 Incident en déblai meuble de type glissement.

Soft ground excavation incident, sliding.
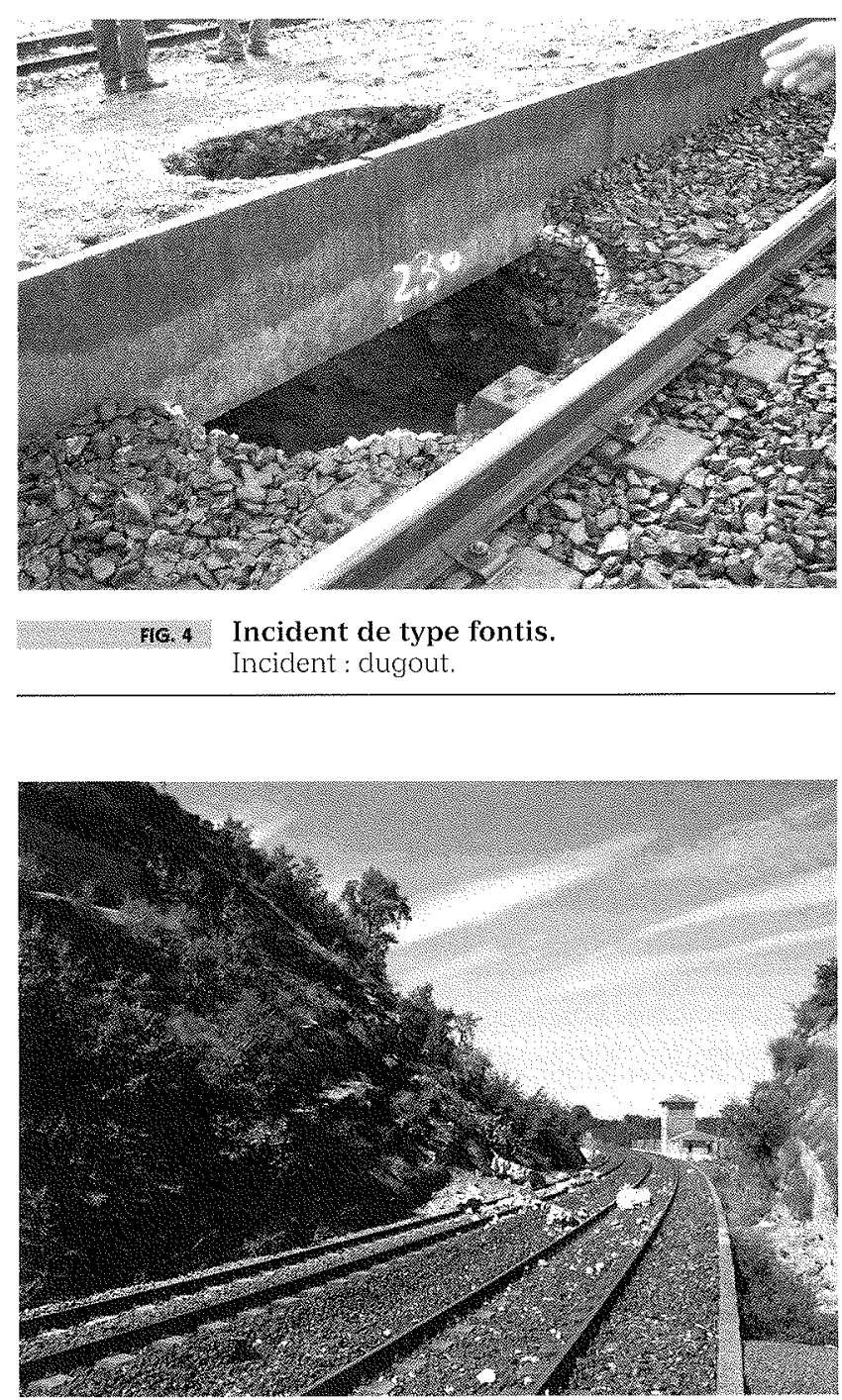

He. 5 Incident en déblai rocheux de type chute de blocs.

Rocky excavation incident, falls of blocks

- l'environnement où l'on doit observer des événements ou des modifications pouvant avoir une influence sur la sécurité des circulations et/ou l'intégrité des infrastructures ferroviaires. ouvrages d'art, fait l'objet de très peu de textes réglementaires et de directives officielles. A ce titre, elle a élaboré ses propres référentiels internes pour garantir au mieux la bonne gestion de cet important patrimoine et, notamment, assurer les exigences de sécurité propres à l'exploitation ferroviaire.

\section{3}

\section{Surveillance des ouvrages en terre}

La surveillance des ouvrages en terre et ouvrages de drainage associés consiste à détecter, répertorier et suivre l'évolution d'indices de désordre ou d'anomalies qui se développent au niveau de ces ouvrages et qui à terme peuvent entraîner des désordres graves. On différencie :

- les emprises ferroviaires qui font l'objet d'une surveillance ;

\section{3}

\section{Classement des ouvrages}

Pour mieux cibler la surveillance et améliorer la maintenance, les ouvrages en terre ont été différenciés : - ouvrages classés « sensibles » : ouvrages en terre qui présentent des désordres ou des indices de désordres, ouvrages ayant fait l'objet d'un traitement qui nécessite un entretien et/ou un suivi et les ouvrages au droit desquels l'environnement a été à l'origine de phénomènes pouvant présenter des risques pour les circulations (chutes de blocs, coulées de boue, crues...) ;

- ouvrages «courants » : ouvrages qui ne présentent pas d'indice recensé ou ouvrages sensibles reclassés en ouvrages courants, après traitement. 
Parmi les 100000 ouvrages en terre du réseau ferré national, le nombre d'ouvrages sensibles est de 8000 unités, très inégalement répartis à travers le réseau. Ces derniers constituent le patrimoine des ouvrages en terre sensibles qui a fait l'objet ces dernières années d'un recensement de la part des services SNCF par recherche d'archives et visites de tronçons de ligne. Ce travail aboutit à l'établissement de fiches signaléticues d'ouvrage comprenant:

- un descriptif de l'ouvrage reprenant le contexte géologique, géotechnique, sa géométrie avec plans et photos, définition des suivis et instrumentations mis en place sur le site instable ;

- un historique des désordres affectant l'ouvrage depuis sa construction et des confortements réalisés pour améliorer sa stabilité (exemple d'éléments d'archives en figure 6).

Il est parfois difficile de remonter très en amont dans la vie de l'ouvrage faute d'archives suffisantes.

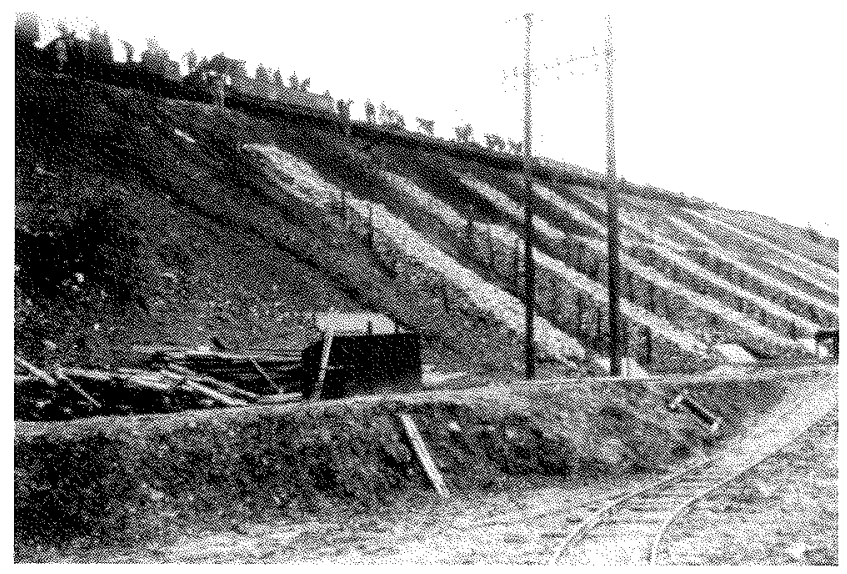

7ic.6. Photo d'archives sur des confortements dans les années $\mathbf{1 9 2 0}$

Archives on reinforcement in the 1920s.

\section{Organisation de la surveillance}

Elle est calée sur l'organisation territoriale du gérant de l'infrastructure (branche infra de la SNCF) et s'appuie sur plusieurs acteurs :

- en établissement «Infrastructure » (structure locale gérant le patrimoine, correspondant géographiquement à une région ou une demi-région administrative) : un correspondant ouvrages en terre, et des agents au sein des unités ( Voie ) chargés de la surveillance au plus près du terrain ;

- au niveau régional au sein du pôle régional ingénierie : un chargé ouvrages en terre spécialiste dans ce domaine, assistant à la gestion du patrimoine des ouvrages en terre des établissements ;

- au niveau national : des experts ouvrages en terre au sein de la direction de l'ingénierie assistant et conseillant les régions et la direction de l'Infrastructure pour les désordres nécessitant un diagnostic pointu ou des études complexes avec moyens de calcul spécialisés (logiciels de calcul de stabilité ou aux éléments finis, etc.). Les mêmes experts, en fonction de l'analyse des risques propres à chaque ouvrage, assistent le maî-

tre d'ouvrage pour le classement technique des opérations de régénération (choix des priorités, échéancier travaux, etc.) en fonction du budget alloué.

Cela nécessite une bonne connaissance du patrimoine des ouvrages en terre et une solide expérience des acteurs. Ceux-ci ont pour mission la réalisation de :

- tournées de surveillance (réalisées essentiellement pour la surveillance de la voie) dont la fréquence varie en fonction des lignes ferroviaires (toutes les 2 à 10 semaines suivant le trafic et l'ancienneté de la voie) qui se font à pied depuis la plate-forme ferroviaire voire en cabine, par les agents de l'unité (Voie » (structure gérant le patrimoine correspondant géographiquement à un département administratif). Dans certains cas l'utilisation de l'hélicoptère permet d'optimiser cette surveillance en intégrant l'ouvrage dans son environnement ;

- visites détaillées au minimum quinquennales, pour suivre l'évolution d'ouvrages en terre sensibles, effectuées par le correspondant OT de l'établissement, avec le chargé ouvrage en terre régional et faisant l'objet de la rédaction d'un procès-verbal, avec propositions de surveillance ou de travaux d'entretien ou de confortement ;

- visites spécifiques intermédiaires pour suivi d'évolution entre deux visites détaillées, par les agents de l'établissement;

- visites spéciales lors d’intempéries (suivant une consigne intempéries rédigée pour chaque établissement et décrivant les points singuliers à observer) ;

- visites d'expertise pour des désordres dont le diagnostic est difficile, ou sur des incidents brutaux qui ont des conséquences sur les circulations ferroviaires, par les experts de la direction de l'Ingénierie en présence des autres acteurs.

La gestion du patrimoine des ouvrages en terre vise à détecter les anomalies au plus tôt, dès leur apparition, pour permettre un bon diagnostic (garant de la sécurité) et l'engagement des mesures utiles avant une évolution irrémédiable. Celui-ci peut s'aider du suivi :

- des défauts géométriques des voies mesurés régulièrement par des engins ferroviaires spécialisés (appelés Mauzin, Mélusine ou IRIS 320) ou menées in situ par l'intermédiaire des reprises de nivellement, lorsque les désordres affectent la plate-forme (voir exemple de graphe de suivi en figure 7)

- de l'instrumentation des ouvrages et/ou de leur environnement, au moyen de :

- tubes inclinométriques (tubes en aluminium scellés dans un forage) permettant de connaître la profondeur de rupture et suivre son évolution (voir graphe de dépouillement en figure 8),

- tubes piézométriques (tubes PVC mis en place dans un forage) permettant de suivre l'évolution du niveau de la nappe,

- suivis topographiques (piquets en fer scellés sur 1,50 m de hauteur) permettant de quantifier les déplacements de l'ouvrage en terre (tassements avec précision de l'ordre du mm et déplacements en planimétrie avec une précision de l'ordre du $\mathrm{cm}$ ),

- témoins de fissuration (sur les ouvrages en terre, les ouvrages d'art ou les parois rocheuses).

En cas d'évolution criticue des mouvements, il peut être mis en place si nécessaire, en attendant un confortement: 

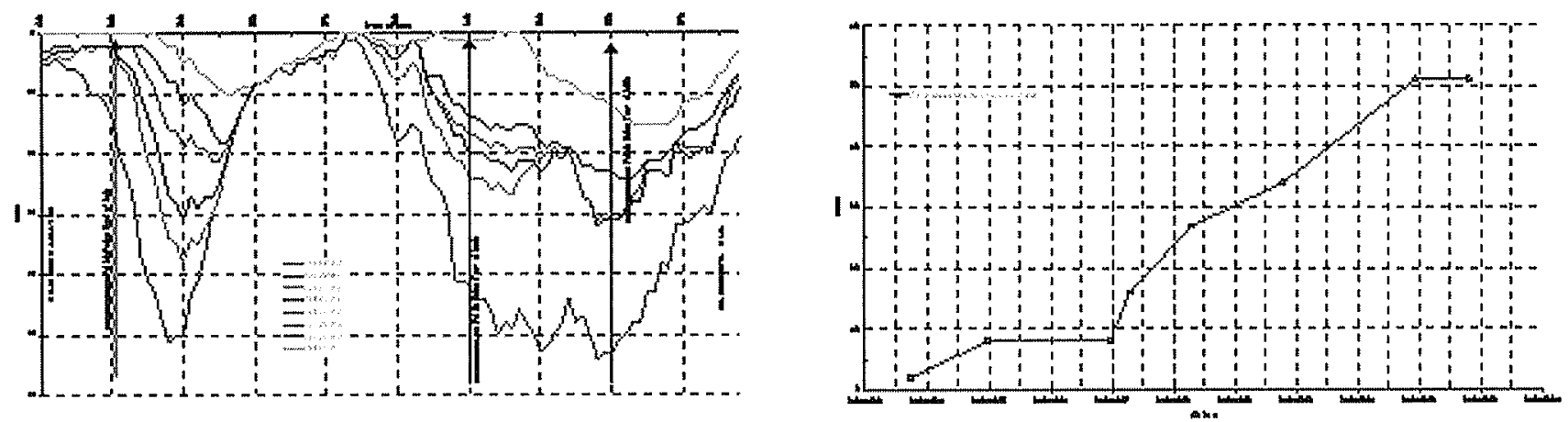

FG. 7 Exemple de graphique de cumulées de reprises de nivellement sur un site instable et évolution en fonction du temps.

Example of leveling recollection on an unstable site and evolution according to time.

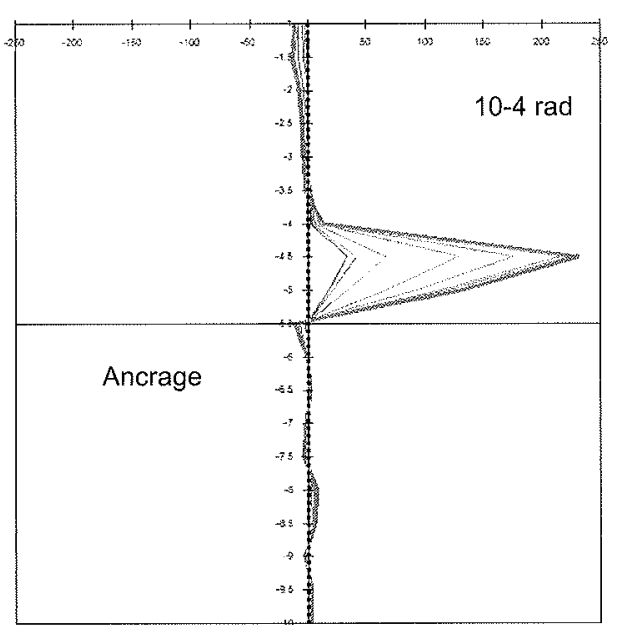

$10-4 \mathrm{rad}$

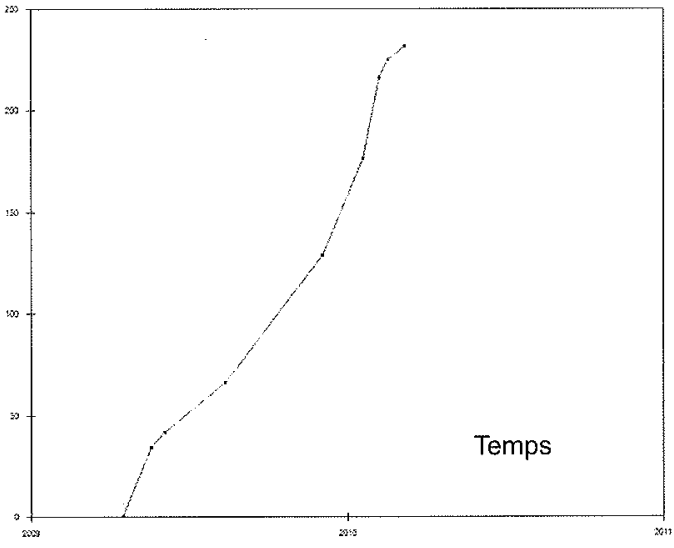

FG. 8 Exemple de dépouillement d'un tube inclinométrique avec logiciel INCLINO 2001 (interne SNCF). A gauche, mesure angulaire ; à droite, évolution en fonction du temps de la mesure angulaire (surface de glissement à 4,50 m de profondeur).

Example of an analysis of an inclinometric tube with software INCLINO on 2001 (SNCF). On the left, angular measure; on the right, evolution according to the time of the angular measure (sliding surface at $4.50 \mathrm{~m}$ of depth).

- un ralentissement voire un arrêt des circulations ;

- une surveillance renforcée ou permanente du site ;

- des mesures confortatives provisoires ;

- des systèmes de détection.

\section{3.}

\section{Traitement des désordres}

Les désordres détectés peuvent nécessiter deux types de traitement:

- des travaux d'entretien (purge de parois rocheuses, curages des drainages, débroussaillages) permettant d'intervenir préventivement avant l'apparition de désordres plus conséquents ;

- des travaux confortatifs pour stabiliser l'ouvrage ou des parades pour se protéger des désordres dont l'origine se situe en dehors des emprises dans l'environnement.

A partir des éléments recueillis (visites d'expertise, sondages de reconnaissance avec essais in situ ou en laboratoire, résultats des suivis topographiques ou inclinométriques, et éléments d'archives), des experts géotechniciens entreprennent l'étude du confortement de l'ouvrage avec, si nécessaire calcul justificatif utilisant les logiciels spécifiques. Pour chaque cas d'ouvrage, on choisit la solution technique la mieux adaptée en fonction du type d'instabilité et des conditions de réalisation (accès, contraintes ferroviaires, contraintes environnementales, etc.) de façon à gêner le moins possible les circulations ferroviaires et à optimiser le projet, tout en garantissant à chaque phase de travaux la sécurité des circulations.

Les travaux de confortement peuvent être très divers en fonction des désordres à traiter.

- Pour les ouvrages meubles, on citera :

- les terrassements consistant à changer la géométrie d'un ouvrage par enlèvement de matériaux (déchargement, reprofilage), par apport de matériaux ou par substitution des terrains en place par des matériaux de meilleure qualité : ces matériaux d'apport ont une granulométrie bien spécifique et proviennent de carrières; on crée ainsi des banquettes de pied et des épaulements pour les remblais (Fig. 9), des masques et des risbermes pour les déblais (Fig. 10) ; 
- les drainages superficiels par fossés (Fig. 11), et profonds par tranchées ou éperons drainants (voir Fig. 9) ;

- les clouages verticaux par pieux battus (type palplanches unitaires, Fig. 12), par pieux forés (de diamètre 200 à $800 \mathrm{~mm}$ ) et par micro-pieux injectés (micropieux type IV) ;

- les clouages inclinés par boulons d'ancrage et peau de confinement en grillage métallique ou géogrille renforcée (Fig. 13) ;

- les soutènements de type berlinoise, gabions métalliques ou béton, mur préfabriqué en L ou mur en poutres ou caissons (technologies très diverses);

- les injections de sols pour traitement des fontis (Fig. 14);

- les protections de surface contre l'érosion (géosynthétiques ou géonattes).
Ces confortements peuvent résulter d'une combinaison de plusieurs types de traitements, dont l'exécution peut parfois être décalée dans le temps (par exemple drainage en. $1^{\text {re }}$ phase, suivi d'un clouage en $2^{\mathrm{e}}$ phase)

- Pour les ouvrages et versants rocheux, on différencie :

- les parades passives (on laisse le phénomène se produire mais on se prémunit des conséquences de la chute) : pièges à cailloux, écrans, merlons, grillage pendu. Lorsque le risque est lié à l'environnement (présence de versants avec chutes de blocs dont l'origine peut être à plusieurs centaines de mètres de la plate-forme ferroviaire), il est mis en place des filets protecteurs (statiques ou dynamiques avec absorption d'énergie) et/ou des filets détecteurs de chutes de rocher, (la détection ayant pour seule fonction d'arrêter les circulations ferroviaires en cas d'éboulement et de

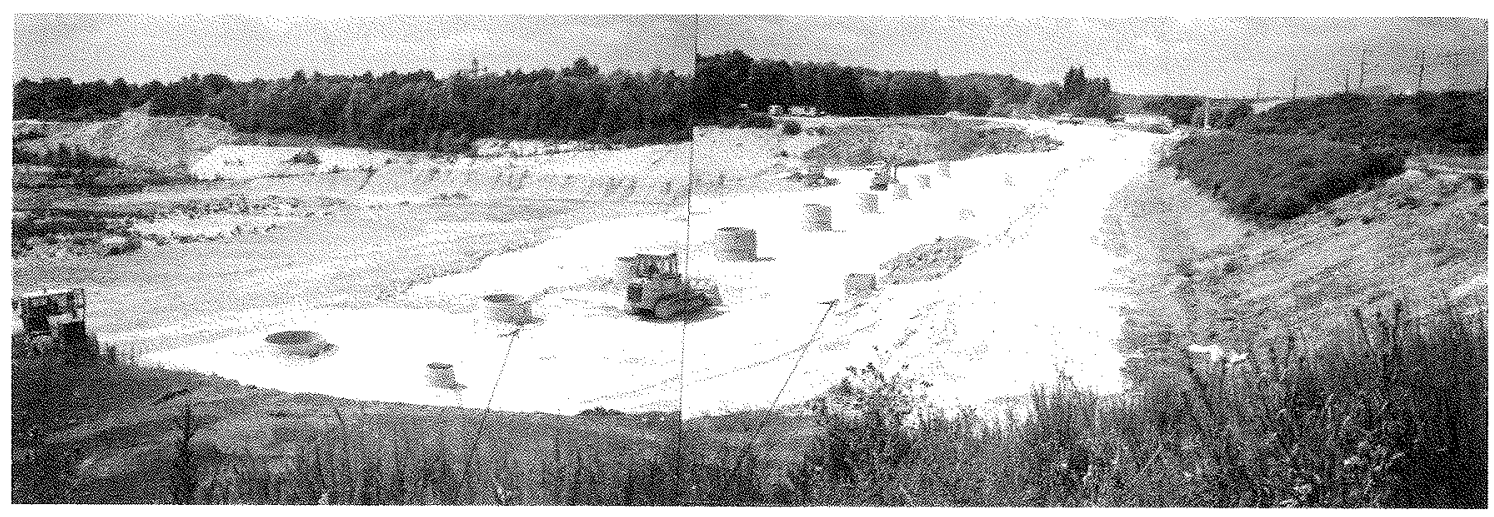

Fr. 9 Confortement d'un remblai par banquette étagée réalisée par plots et par tranchée et puits drainants.

Embankment reinforcement by staged banquette realized by plots and by trench and draining wells.

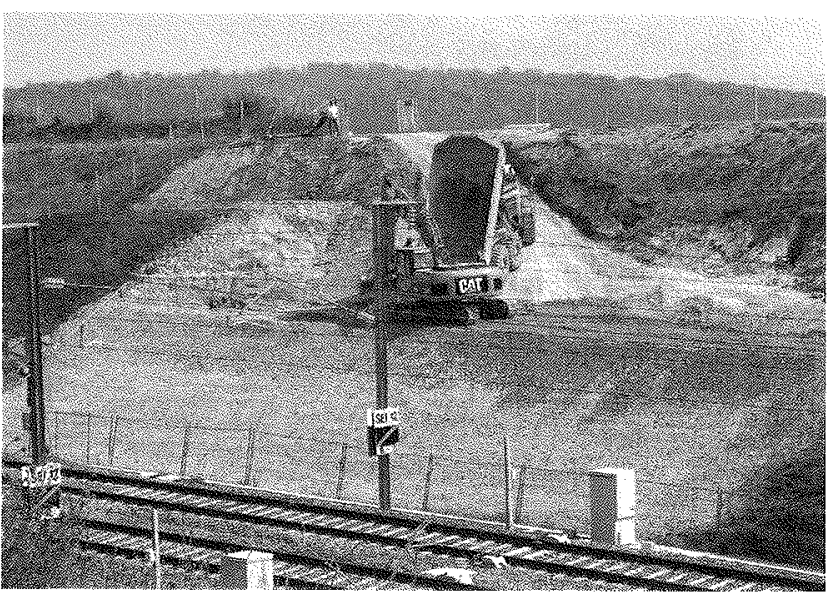

fis 10 Mise en œuvre d'un masque en déblai. Realization of a mask in excavation.

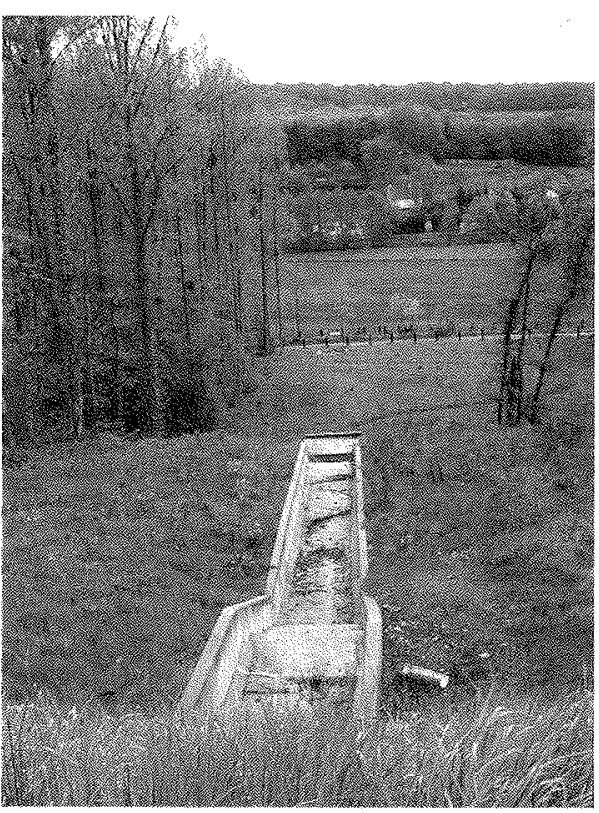

16. 11 Exutoire d'un drainage transversale de remblai. Release of a transversal drainage in embankment. 


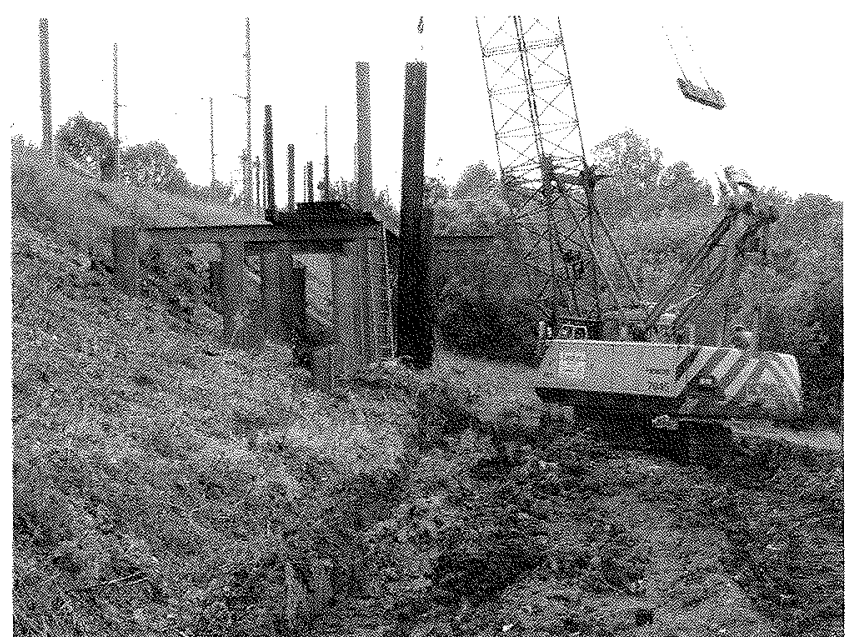

2. IG 12 Clouage par palplanches unitaires. Nailing by unitaries « palplanches ».

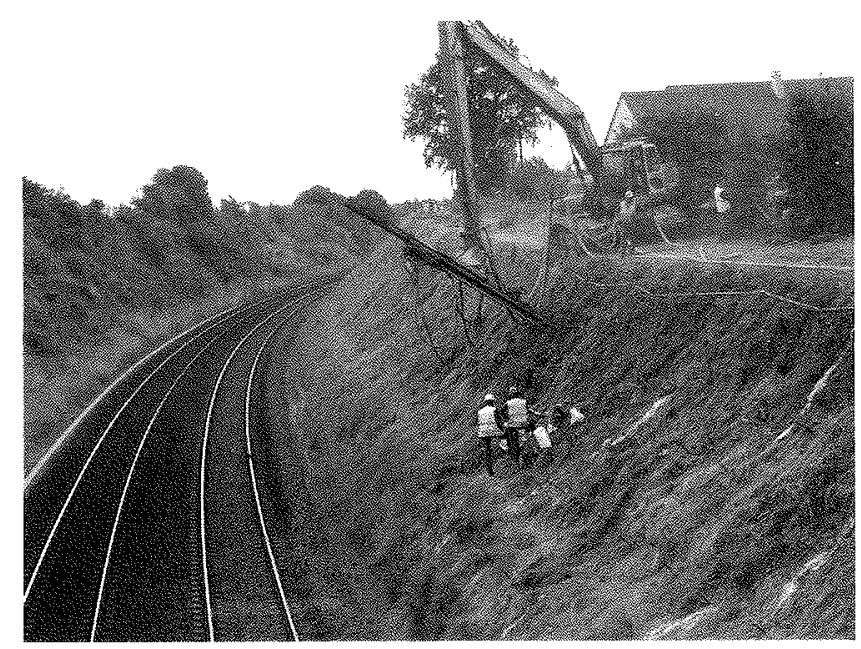

F6. 13 Clouage incliné d'un talus de déblai. Oblique nailing of an excavation slope. rupture d'un fil : transmission d'une alerte, mise au rouge de la signalisation, arrêt des convois avant la zone critique) ;

- les parades actives (on s'oppose à la chute) : ancrages, grillages plaqués-ancrés (Fig. 15), emmaillotages par filets de câbles (Fig. 16) butons, ouvrages en béton, béton projeté, réservés le plus souvent au traitement des tranchées rocheuses proches des voies.

Les travaux sont réalisés par des entreprises extérieures sous maîtrise d'cuvre de l'établissement qui nomme un chef de projet, et avec l'assistance du chargé des ouvrages en terre régional et éventuellement la présence des experts nationaux pour des travaux plus pointus (confortements rocheux complexes, clouage vertical ou incliné, voire des techniques innovantes) ou des aléas de chantier.

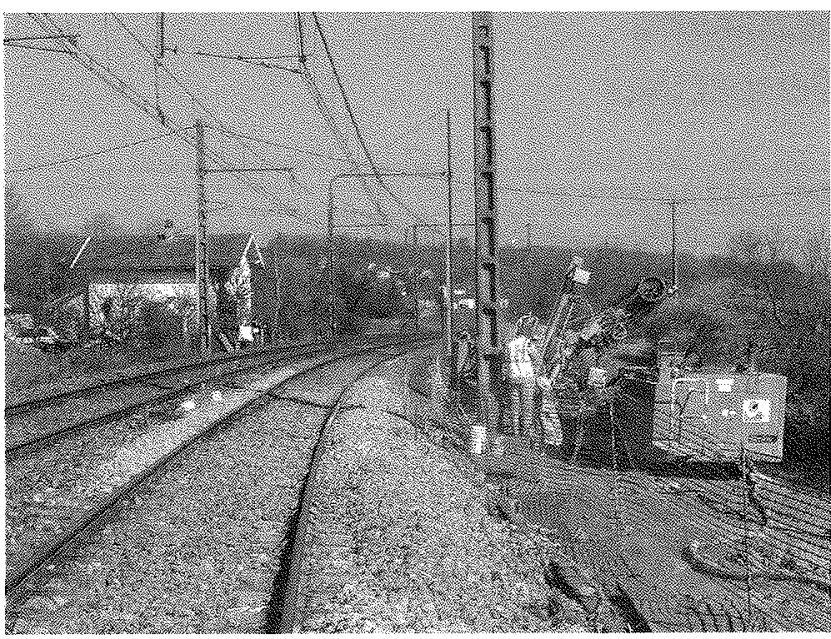

fic. 14 Travaux d'injection de sols (forages biais pour ne pas gêner les circulations.

Injection works of grounds (oblics drillings not to bother traffic)

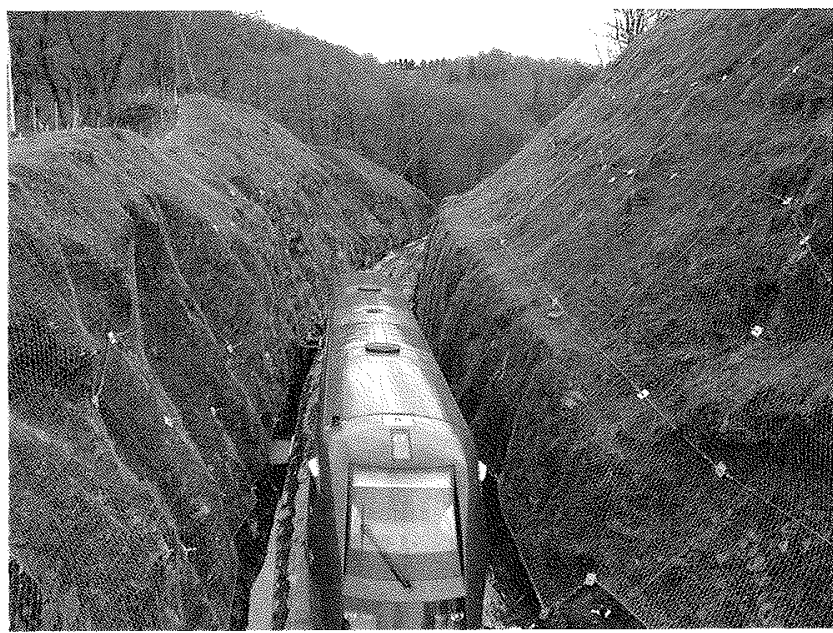

Fic is Revêtement grillagé sur une tranchée rocheuse.

Cover fenced in on a rocky trench.

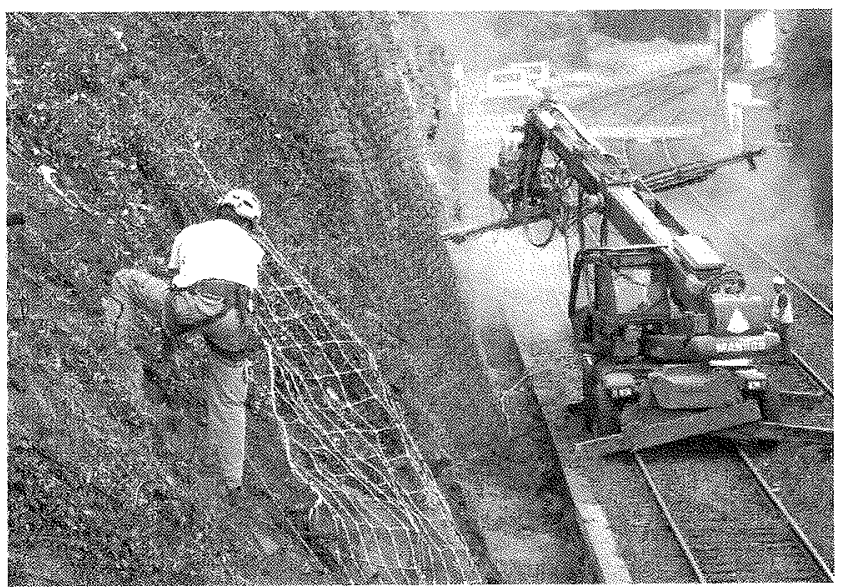

416. 46. Réalisation d'ancrages et mise en place de filets de câbles sur une tranchée rocheuse. Realization of anchorings and implementation of nets of cables on a rocky trench. 


\section{Perspectives}

L'amélioration de la maintenance des ouvrages en terre est axée sur :

- le développement d'un fichier informatisé national regroupant l'ensemble des ouvrages en terre sensibles pour le compte du maître d'ouvrage RFF. Il permet de mieux cibler les ouvrages à traiter en priorité (traitement préventif de l'ensemble d'une zone plutôt que plusieurs traitements curatifs sur plusieurs années à chaque incident) ;

- la réalisation de retours d'expérience et de synthèses nationales sur différents thèmes (corrélations entre défauts d'entretien et incidents, ouvrages rocheux, relations incidents/pluviométrie...);

- la mise en ceuvre d'une veille technologique (recherche de techniques innovantes) ;

- la multiplication de thèmes de recherche, en liaison avec des organismes publics ou privés (comportement des limons du Nord de la France, méthodes de reconnaissance géophysique, détection de chutes de rochers, fibre optique, télémesures et télésurveillance, etc.) ou en interne SNCF.

\section{Conclusion}

Il a été montré tout au long de cet article l'importance de la stabilité et/ou de la protection des Ouvrages en terre tant pour la sécurité et la régularité des circulations ferroviaires que pour la fiabilité des itinéraires. Ces objectifs fondamentaux nécessitent une gestion spécifique du patrimoine des ouvrages en terre. Cette gestion est difficile et délicate du fait de leur extension et de leur grande variété tant géométricque que géotechnique, et d'autre part, de leur grande sensibilité aux aléas climatiques. C'est un patrimoine non figé dont le nombre évolue en permanence. La SNCF pour le compte de RFF met en cuvre une méthodologie de surveillance et d'intervention spécifique visant à détecter au plus tôt les indices de désordres pour pouvoir les traiter préventivement et limiter ainsi les conséquences sur les circulations ferroviaires. Ces missions requièrent des compétences spécialisées particulières au sein d'une organisation spécifique, cumulant les expériences et qui n'a pas d'équivalent dans le domaine routier. 